\title{
Morphological transformations based on optical symbolic substitution and polarization-encoded optical shadow-casting systems
}

\author{
Abdallah K. Cherri \\ Department of Electrical and Computer Engineering, The University of Michigan-Dearborn, \\ 4901 Evergreen Rd, Dearborn, MI 48128, USA \\ Abdul Ahad S. Awwal \\ Computer Science and Engineering Department, Wright State University, Dayton, OH 45435, USA
}

and

Mohammad A. Karim

Electrical Engineering Department, The University of Dayton, 300 College Park Ave, Dayton, OH 45469-0001, USA

Received 12 September 1990

\begin{abstract}
By combining two powerful optical computing techniques, namely, optical symbolic substitution (OSS) and polarization-encoded optical shadow-casting (POSC), morphological or shape transformation operations are demonstrated. Accordingly, erosion, dilation, opening, and closing operations are realized using both OSS and POSC schemes. These morphological operations are used for noise removal in binary images.
\end{abstract}

\section{Introduction}

The optical symbolic substitution (OSS) is a pattern transformation technique which realizes digital logic optically for information processing in two-dimensional images [1]. In this scheme, data are encoded as spatial patterns and operators are seen as pattern transformation rules $[2,3]$. In its operation, OSS functions by mapping a to-be-recognized pattern (recognition phase) into a new pattern (substitution phase). Because of its extensive parallelism as well as spatial orientation, OSS appears particularly attractive for implementing image processing techniques. Optical symbolic substitution based edge detection [4,5], histogram equalization [6], morphological operations [7], image skeletonization [8], as well as arithmetic and/or logical operations [914] have already been realized. The OSS technique consists of a series of image duplication, image shift- ing, and image overlapping in a particular and sequential order. By examining many of these SS architectures, it has been shown that any SS logic can be expressed as a sum of product (SOP) operations $[15,16]$.

On the other hand, optical shadow-casting (OSC) is another powerful optical scheme which utilizes light emitting diodes (LEDs) array to compute combinatorial logic function [17]. In OSC, inputs are spatially encoded 2D binary pixel pattern composed of transparent and opaque, vertical and horizontal polarization. The encoded inputs are placed in perfect contact at the input plane resulting in an input overlap plane. The input overlap pixel is illuminated by a set of spatially displaced LEDs resulting in an overlap of projected shadows. A decoding mask placed at the output plane detects the output. It has been shown that OSC is capable of performing any logical operation of two or more variables by chang- 
ing the LED pattern or encoding $[18,19]$. In other words, a particular combination of input is selected by means of encoding and/or LED pattern and then a single [18] or multiple [19] outputs are substituted. Consequently, OSC can also be classified as a symbolic substitution technique. In its operation, we have shown [16] that, in an OSC scheme, the recognition and substitution phases take place by means of LEDs projecting shadows of the overlap pattern to the output plane. The location and the types of vertical $[20,21]$ LEDs are chosen such that the transparent openings can project light in specific locations in the output plane. In other words, LEDs are chosen to cause predetermined shifts and overlap of the shadows of the input overlap plane. Therefore, the OSC scheme can be seen as operating in a similar fashion as the OSS scheme. More interestingly, one can perform any symbolic substitution logic using optical shadow-casting scheme [22].

In this communication, by combining the strengths of both OSS and OSC schemes, morphological transformations are demonstrated. Accordingly, morphological operations such as erosion, dilation, opening, and closing are realized using both OSC and OSS schemes.

\section{Morphological transformations}

The image processing technique of shrinking and expanding a binary image to reduce or remove unwanted artifacts is very old but is still used. A single pixel shrink and expand operation, referred to as mathematical morphology [23] will be enough for cleaning an image when artifacts are small. When artifacts are large, however, several iterations may be necessary for sufficient image smoothing.

Algebraically, morphological analysis involving images consists of two basic set operations. Minkowski addition and subtraction. The image $X$ can be conceived as an ensemble of black pixels constituting the object and white pixels constituting the background. The operations involve a specific binary operating image called a structuring element. The structuring element $E$ determines the pixels of $X$ that will be affected by the transformations.

The Minkowski sum or dilation is found by placing the reference pixel (origin) of the structuring ele- ment $E$ over each of the activated pixels (those having a value of 0 ) of $X$ and then taking the union of all the resulting copies. On the other hand, Minkowski subtraction or erosion can be described in terms of the structure element translation as follows. The origin of the structuring element $E$ is placed successively over each activated pixel of $X$. If, for a given pixel, say $(i, j)$, the activated pixel of the translated copy of $E$ is a subimage of $X$, then the $(i, j)$ pixel value is set to 0 ; otherwise, the $(i, j)$ pixel is set to 1 in the eroded image. Two other operations that follow directly from dilation and erosion are opening and closing. A pixel is activated in the opened image if and only if it is a part of a fitted copy of the structuring element. Opening is achieved by having an erosion operation followed by a dilation. On the other hand, closing consists of a dilation followed by an erosion [23].

The four morphological operations can be efficiently implemented using OSS based on POSC. Fig. 1 describes the necessary SS rules for implementing these operations for a specific structuring element. To perform an erosion, one needs to replace every occurrence of the structuring element (the recognition pattern ) in the input image with a single opaque pixel (the substitution pattern). The dilation can also be achieved using SS rules by replacing every opaque pixel of the input image by the structuring element. Again, by combining erosion and dilation, the opening and closing of an image are realized.

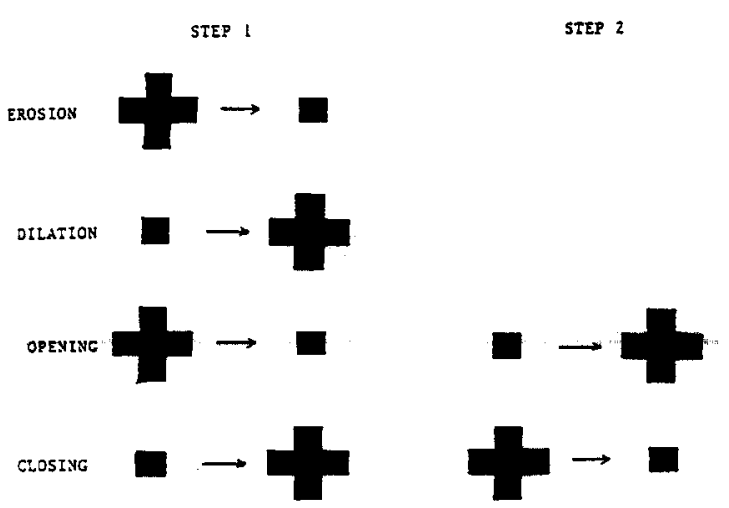

Fig. 1. Symbolic substitution rules for morphological transformations. 


\section{Optical symbolic substitution based OSC system}

Optical SS scheme for realizing morphological transformation can be realized using phase-only holograms and classical optical elements (e.g., mirrors, prisms, etc. ) [7]. The basic system performs a series of operations in the following order: (a) splitting an input image, (b) shifting split images, (c) superimposing the shifted an unshifted images, (d) regenerating the superimposed image, (e) splitting the regenerated image, (f) shifting the resulting images, and $(g)$ superimposing the shifted images.

However, optical shadow-casting scheme provides faster image replication and spatial shifting. By choosing the spacing between the LEDs and distance from the source plane to the input plane and from the input plane to the output plane [17], replicated and shifted copies of the input plane are obtained superimposed on the screen. The source LEDs and their ON-OFF states (representing the structure element $E$ ) determine the number of copies and their relative shifts. Fig. 2 shows a recognition unit using intensity coded binary logic where 0 and 1 are, respectively, represented as opaque and transparent. To meet the requirement of having a cross-shaped structuring element (fig. 1), for instance, five LEDs $\{B, D, E, F$, and $H\}$ of the source plane (denoting the five opaque pixels of the structural element) are turned $\mathrm{ON}$ accordingly. This configuration of LEDs yields five shifted and superimposed copies of the input image. The first, second, third, fourth, and fifth copies are, respectively, shifted down, shifted up, not shifted, shifted left, and shifted right by one pixel. The overlapping of these copies produces the output image. Obviously, this OSS based OSC system actually realizes an erosion operation of the input binary image with a cross-shaped structuring element. Note that by turning ON-OFF different LEDs at the source plane, erosion operation by many other structural elements is possible.

On the other hand, fig. 2 can be also used to achieve a dilated image with the cross-shaped structuring element. The input image, in this case, is first complemented and then directed to the input plane of the OSC unit where again five copies of the inverted input image are obtained. Shift operations similar to those encountered earlier are realized and the overlapped copies consist the partial output. Finally,

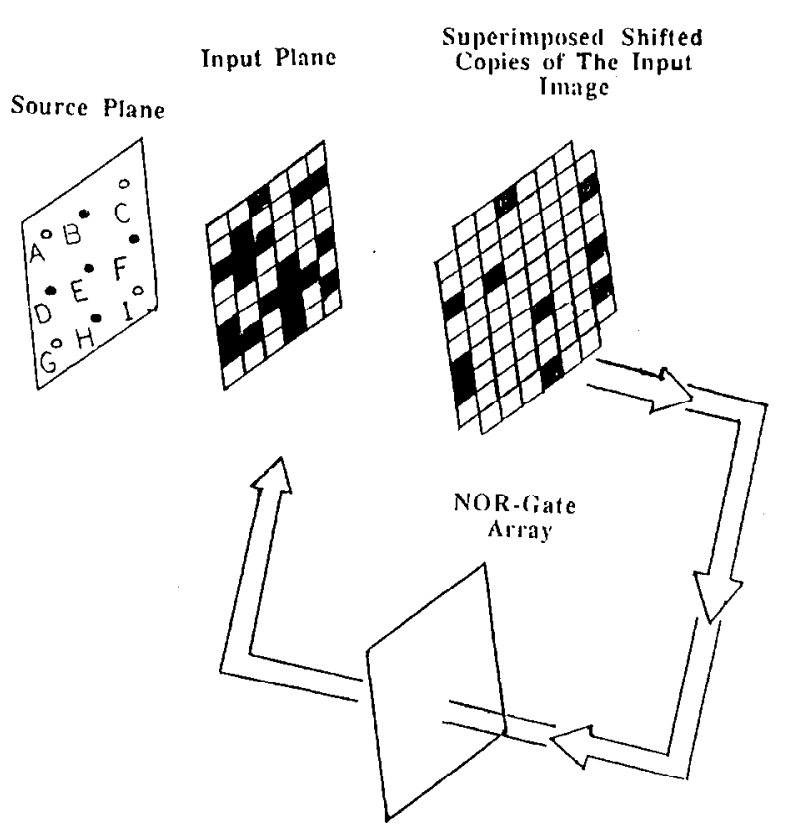

Fig. 2. Optical symbolic substitution based optical shadow-casting system for morphological operations.

the partial output image is incident on a NOR-gate array whose output is the inversion of the partial output image. Therefore, the SS based OSC unit shown can be used for both erosion and dilation operations provided that in the latter case, the input image and the resulting output image are inverted using a NOR-gate array. Further, by adjusting the LEDs at the source plane, many structure elements of any desired shape may be implemented. Furthermore, this system can be used to execute iteratively any morphological operation by simply feeding back the resulting output image and reintroducing it to the input of the system either inverted through a NORgate array or directly without inversion. Consequently, several morphological operations can be carried out sequentially one after the other, i.e. erosion followed by dilation followed by erosion, etc.

Now, since opening is equivalent to an erosion followed by a dilation, then the output of the system of fig. 2 is reintroduced (after inversion) as an input to the system. Similarly, one can obtain closing operation from the system by executing a dilation followed by an erosion operation.

For illustration purposes, computer simulation re- 


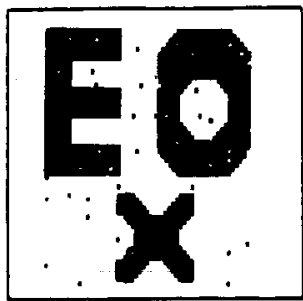

(a)

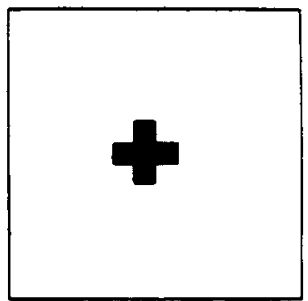

(b)
Fig. 3. (a) Original image and (b) the structuring element.

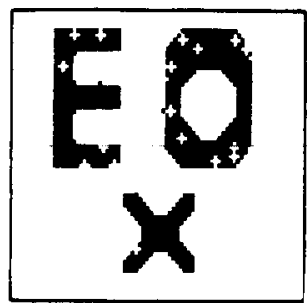

(a)

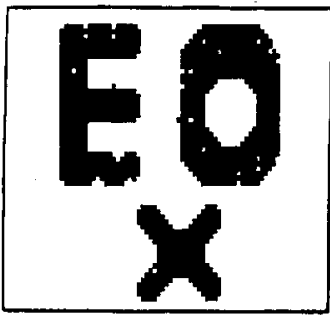

(c)

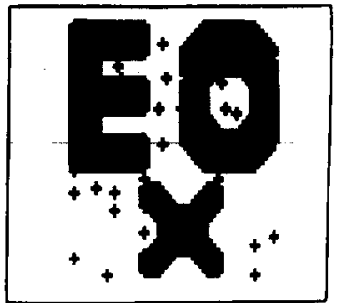

(b)

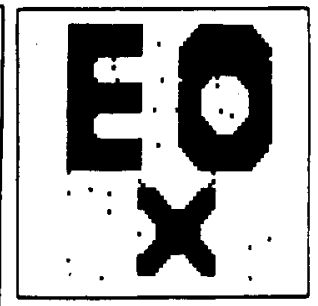

(d)
Fig. 4. Morphological transformations: (a) eroded image, (b) dilated image, (c) opened image, and (d) closed image.

sults will be presented where a noisy binary image as shown in fig. 3a is subjected to morphological transformations by the structuring element shown in fig. 3b. Fig. 4a shows the eroded image where the objects in the image have been reduced in size. Also, note that much of the "pepper" noise has been eliminated while at the same time the "salt" noise has been increased. Actually, every isolated white pixel takes the shape of the structuring element. Fig. $4 \mathrm{~b}$ shows the dilated image. Here, the sizes of the objects in the image have increased, and in contrast to the eroded image, most of the "salt" pixels have been removed while the "pepper" pixels have increased in size and have taken the shape of the structuring element. Fig. $4 \mathrm{c}$, on the other hand, shows the opened image. Note that in this figure the isolated "pepper" pixels and some of the "salt" pixels have been eliminated. Finally, fig. $4 \mathrm{~d}$ shows the closed image. Note that the holes inside the image have been filled. It is obvious that an erosion of this output would eliminate all of its isolated black pixels. It is worth mentioning that the results of morphological operations are extensively dependent on the shape of the structuring element.

\section{Conclusion}

In this paper, we have extended and combined the capabilities of the two powerful techniques of OSS and OSC to realize morphological operations. A simple OSC system combined with a NOR-gate array can be organized to generate the four basic morphological operations. This novel OSS based OSC system, in addition to its simplicity, has the potential to execute and implement many optical parallel architectures in the area of optical computing and optical information processing.

\section{References}

[1] A. Huang, Tech. Digest of IEEE 10th International optical Conference, 1983, p. 13.

[2] K.H. Brenner, A. Huang and N. Streibel, Appl. Optics 25 (1986) 3054.

[3] K.H. Brenner, Appl. Optics 25 (1986) 3061.

[4] A.K. Cherri and M.A. Karim, Appl. Optics 28 (1989) 4644.

[5] A.K. Cherri and M.A. Karim, Optics Comm. 74 (1989) 10.

[6] A.K. Cherri and M.A. Karim, Opt. Eng. 28 (1989) 638.

[7] A.K. Cherri and M.A. Karim, Microwave and Optical Technology Letters 2 (1989) 282.

[8] G. Eichmann, J. Zhu and Y. Li, Appl. Optics 27 (1988) 2905.

[9] Y. Li and G. Eichmann, Appl. Optics 26 (1987) 2328.

[10] Y. Li, G. Eichmann, R. Dorisinville and R.R. Alfano, Optics Comm. 63 (1987) 375.

[11] A.K. Cherri and M.A. Karim, Appl. Optics 27 (1988) 3824.

[12] A.K. Cherri and M.A. Karim, Optics Comm. 70 (1989) 455.

[13] E. Rotha, D. Casasent and E. Barnhard, Appl. Optics 27 (1987) 817.

[14] J.N. Mait and K.H. Brenner, Appl. Optics 27 (1988) 1692.

[15] E.L. Johnson and M.A. Karim, Digital design; a pragmatic approach (PWS publishers, Boston, 1987).

[16] A.A.S. Awwal, A.K. Cherri and M.A. Karim, SPIE Proceeding 1215 (1990) 506.

[17] J. Tanida and Y. Ichioka, J. Opt. Soc. Am. 73 (1983) 800. 
[18] M.A. Karim, A.A.S. Awwal and A.K. Cherri, Appl. Optics 28 (1987) 2720.

[19] A.A.S. Awwal and M.A. Karim, Appl. Optics 27 (1988) 932.

[20] A.A.S. Awwal, M.A. Karim and A.K. Cherri, Appl. Optics 26 (1987) 4814.
[21] A.A.S. Awwal and M.A. Karim, Appl. Optics 28 (1989) 284. [22] A. Louri, Appl. Optics 28 (1989) 3264.

[23] J. Serra, Image analysis and mathematical morphology (Academic Press, London, 1982). 